\title{
Evaluating the effects of cardiac resynchronization therapy on pathophysiological pathways of heart failure using surrogate biomarkers
}

\author{
Hamza Sunman ${ }^{1}$, Adem Özkan², Hikmet Yorgun ${ }^{3}$, Uğur Canpolat ${ }^{3}$, \\ Erdem Karabulut ${ }^{4}$, Tülin Bayrak ${ }^{5}$, Ergün Barış Kaya ${ }^{3}$, Lale Tokgözoğlu ${ }^{3}$, \\ Necla Özer ${ }^{3}$, Asuman Özkara ${ }^{6}$, Kudret Aytemir ${ }^{3}$, Ali Oto ${ }^{3}$ \\ ${ }^{1}$ Ministry of Health Dışkapı Yıldırım Beyazıt Research and Educational Hospital, Turkey \\ ${ }^{2}$ Public Health Laboratory, Erzurum, Turkey \\ ${ }^{3}$ Department of Cardiology, Hacettepe University, Ankara, Turkey \\ ${ }^{4}$ Department of Biostatistics, Hacettepe University, Ankara, Turkey \\ ${ }^{5}$ Department of Biochemistry, Ordu University, Ordu, Turkey \\ ${ }^{6}$ Department of Biochemistry, Hacettepe University, Ankara, Turkey
}

\begin{abstract}
Background: Several studies have investigated the effects of cardiac resynchronization therapy (CRT) on heart failure (HF), but none have evaluated the pathophysiological pathways involved in a single group of patients. Therefore, this study aims to assess the long-term effects of CRT on six different pathophysiological pathways involved in the process of HF by the use of surrogate biomarkers.

Methods: In a group 44 patients with HF, six groups of biomarkers were measured, both at baseline and 1 year after CRT implantation: inflammation (interleukin [IL]-4, IL-6, tumor necrosis factor [TNF]- $\alpha$, high sensitive C-reactive protein [hsCRP]); oxidative stress (myeloperoxidase [MPO], oxidized low-density lipoprotein [oxLDL], uric acid); extracellular matrix (ECM) remodeling (matrix metalloproteinase [MMP]-2 and -9, galectin-3, procollagen III N-terminal propeptide [prokol-3NT]); neurohormonal pathways (endothelin-1, chromogranin-A); myocyte injury (troponin T, creatine kinase $M B$ fraction [CK-MB]), myocyte stress (B-type natriuretic peptide [BNP]). CRT responders were defined as patients with $\geq 15 \%$ reduction in left ventricular end-systolic volume at 12 months post-CRT. Results: At 1-year follow-up, 72.7\% ( $n=32)$ of the patients were categorized as CRT responders. In these patients, the levels of IL-6, MPO, oxLDL, MMP-2, galectin-3, troponin T, and BNP were significantly reduced as compared to baseline values. While the biomarkers for myocyte stress (effect size $=0.357$; $p=0.001$ ), ECM remodeling (effect size $=0.343 ; p=0.015$ ) and oxidative stress (effect size $=0.247$; $p=0.039)$ showed a significant change in the CRT responders during follow-up, the biomarkers for other pathophysiological pathways did not show a significant alteration.

Conclusions: In the present study, a significant reduction was only observed in the biomarkers of myocardial stress, ECM remodeling, and oxidative stress among all the CRT responder subjects. (Cardiol J 2018; 25, 1: 42-51)
\end{abstract}

Key words: cardiac resynchronization therapy, responder, pathophysiology, biomarkers

\section{Introduction}

Heart failure (HF) is a progressive, chronic myocardial disorder involving various pathophysi- ological pathways. It has recently been reported that six major pathways are involved, both in the development and the progression of HF [1]. Various management strategies including medical treat-

Address for correspondence: Dr. Hamza Sunman, Ministry of Health Dışkapı Yıldırım Beyazıt Research and Educational Hospital, Turkey, e-mail: hamzasunman@gmail.com 
ment and/or device implantation are aimed at either slowing down or reversing the disease process. Cardiac resynchronization therapy (CRT) is a valuable interventional therapeutic option in patients with systolic HF and left ventricular (LV) conduction disturbances, as it decreases the chances of both morbidity as well as mortality [2,3]. Yet, up to $30-40 \%$ of the patients do not benefit from CRT implantation for several reasons [4, 5]. Although several studies using circulating biomarkers have been carried out to identify patient responsiveness to CRT [6-10], none could assess all the predefined pathophysiological pathways as a whole in the same patient population. Hence, the aim undertaken was to evaluate the relationship of CRT responsiveness with six different pathophysiological pathways of HF using surrogate biomarkers.

\section{Methods}

A total of 44 patients diagnosed with systolic HF, who underwent CRT implantation were included in the study. The inclusion criteria were as follows: New York Heart Association (NYHA) class II-III symptoms despite optimal tolerable medical therapy, $\mathrm{LV}$ ejection fraction of $\leq 35 \%$, and a QRS duration of $>120 \mathrm{~ms}$ either resulting from bundle branch block or intraventricular conduction delay. All subjects included in the study had to meet the inclusion criteria. Patients were excluded if they had atrial fibrillation (AF), acute or chronic inflammatory or infectious disease, recent ( $\leq 30$ days) clinical diagnosis of acute coronary syndrome, acute decompensated HF, or had recently $(<6$ months) undergone a coronary revascularization procedure or a coronary by-pass surgery. The recorded baseline characteristics included patient demographics, QRS duration, etiology of cardiomyopathy, medical history in relation to hypertension, diabetes, smoking, AF, and the use of the medications prescribed including beta-blockers, angiotensin-converting enzyme inhibitors, spironolactone, digoxin, and diuretics. The study protocol was approved by the Ethics Committee. Written informed consent was obtained from all patients.

Serial echocardiography was performed at both times, before and after CRT implantation in order to assess the degree of $\mathrm{LV}$ reverse remodeling and changes in cardiac function. LV end-systolic volume, LV end-diastolic volume, and ejection fraction were assessed using Simpson's equation. The severity of mitral regurgitation was graded semi-quantitatively by color flow Doppler in the conventional parasternal long axis and by the images of the apical 4-chambers. Living patients were classified as CRT responders if they showed $a \geq 15 \%$ decrease in LV end-systolic volume (compared to baseline) at the 12-month follow-up [11].

The LV lead was placed in the coronary sinus in order to achieve permanent epicardial stimulation as described earlier [12]. CRT device and lead placements were accomplished in all the subjects without major complications (Medtronic, St. Paul, MN, USA and Biotronik, Berlin, Germany). The pacing mode was set at DDD which is programmed to maximize biventricular pacing, and the atrioventricular and interventricular delays were optimized using Doppler echocardiography. Coronary sinus lead position, pacing mode, and the programming of timing intervals were evaluated at $1,3,6$, and 12 months after CRT implantation.

Blood samples were obtained from the patients after a 30 -min bed rest, both before CRT and at the 12 -month follow-up visit and were stored at $-80^{\circ} \mathrm{C}$ until used. Biomarkers that played a role in the pathogenesis of HF were classified into six groups on the basis of the data obtained from previous studies [1]. The biomarkers that were assessed included interleukin (IL)-4 (BMS225INST, Bendermed, Austria), IL-6 (BMS213INS, Bendermed, Austria), tumor necrosis factor-alpha (TNF- $\alpha$ ) (BMS223INST, Bendermed, Austria) and high-sensitive $\mathrm{C}$-reactive protein (hsCRP) (EIA3954, DRG, Austria) for inflammation; oxidized low density lipoprotein (oxLDL) (K7810, Immune Diagnostic, Germany), myeloperoxidase (MPO) (K6631, Immune Diagnostic, Germany) and uric acid (routine) for oxidative stress; matrix metalloproteinase (MMP)-2 (ELH-MMP2-001, RayBiotech, Austria), MMP-9 (BMS2016/2, Bendermed, Austria), galectin-3 (BMS279/2, Bendermed, Austria) and procollagen III N-terminal propeptide (prokol--3NT) (E0573Hu, Uscnk, Austria) for extracellular remodeling; the neurohormones endothelin-1 (BI20052, BIOMEDICA, Canada) and chromogranin-A (RSCYK070R, Biovendor, Germany) by ELISA; troponin $\mathrm{T}$ (routine), creatine kinase $\mathrm{MB}$ fraction (CK-MB) (routine) for myocyte damage; and B-type natriuretic peptide (BNP) (routine) for myocyte stress. Blood samples from all patients were processed according to instructions given by the manufacturer and were spectrophotometrically read on a SpectraMax M2 reader (Molecular Devices, Inc., Silicon Valley, CA, USA).

\section{Statistical analysis}

All statistical analyses were performed using SPSS for Windows (ver. 20, IBM SPSS Inc., Chicago, IL). A p-value $<0.05$ was considered to 
Table 1. Baseline characteristics of study participants.

\begin{tabular}{lcccc}
\hline & $\begin{array}{c}\text { All patients } \\
(\mathbf{n}=\mathbf{4 4 )}\end{array}$ & $\begin{array}{c}\text { Responders } \\
(\mathbf{n}=32)\end{array}$ & $\begin{array}{c}\text { Non-responders } \\
\text { (n = 12) }\end{array}$ & P \\
\hline Age [years] & $61.93 \pm 11.81$ & $63.13 \pm 10.4$ & $59.0 \pm 14.8$ & 0.293 \\
Gender (female) & $15(33.3 \%)$ & $11(34.4 \%)$ & $4(30.8 \%)$ & 0.816 \\
NYHA class & $2.88 \pm 0.26$ & $2.83 \pm 0.3$ & $3.0 \pm 0.0$ & 0.003 \\
Hypertension & $34(75.6 \%)$ & $22(68.8 \%)$ & $12(92.3 \%)$ & 0.096 \\
Diabetes mellitus & $10(22.2 \%)$ & $3(9.4 \%)$ & $7(53.8 \%)$ & 0.003 \\
Heart failure etiology: & & & 0.307 \\
$\quad$ Non-ischemic & $27(61.4 \%)$ & $22(68.8 \%)$ & $5(41.7 \%)$ & \\
$\quad$ Ischemic & $17(38.6 \%)$ & $10(31.3 \%)$ & $7(58.3 \%)$ & 0.059 \\
QRS duration [ms] & $159.82 \pm 26.84$ & $164.6 \pm 26.6$ & $148.0 \pm 24.6$ & $9(69.2 \%)$ \\
Left bundle branch block & $41(91.1 \%)$ & $32(100 \%)$ & $111.43 \pm 43.05$ & 0.537 \\
End-diastolic volume index $\left[\mathrm{mL} / \mathrm{m}^{2}\right]$ & $105.56 \pm 38.04$ & $103.36 \pm 36.48$ & $81.54 \pm 66.58$ & 0.590 \\
End-systolic volume index $\left[\mathrm{mL} / \mathrm{m}^{2}\right]$ & $77.48 \pm 30.13$ & $75.96 \pm 29.91$ & $26.9 \pm 2.6$ \\
Ejection fraction [\%] & $26.98 \pm 4.79$ & $27.0 \pm 5.5$ & 0.950 \\
\hline
\end{tabular}

Values are presented as mean \pm standard deviation or number (percentage); NYHA - New York Heart Association

be statistically significant. Quantitative variables with normal distribution were represented as mean \pm standard deviation and were analyzed by the Student t-test; whereas non-normally distributed variables were represented as median-interquartile range (IQR) and were analyzed using the twotailed Mann-Whitney U-test. Categorical data were expressed as proportions and compared using $\chi^{2}$ test. Initially, a univariate analysis was performed at baseline levels to investigate the association between biomarkers and the incidence of CRT-response. Subsequently, a multivariate analysis (backward LR) was also performed including those variables which had a $p$-value $\leq 0.25$ in the univariate analysis. The receiver-operating characteristic (ROC) curve was used to evaluate the performance of biomarker levels to predict the response to CRT. An optimal cut-off point was chosen as the sensitivity and specificity were maximized. The correlation coefficients that were reported were based on a non-parametric method (Spearman rank). Timerelated changes in the biomarkers were analyzed by paired t test or Wilcoxon test. 'Repeated measures two-way ANOVA' was used to compare the change in biomarkers after CRT, both individually and as a group. Univariate and multivariate partial Eta values that indicate the degree of relationship between the disease status and changes in biomarkers were obtained from ANOVA tables, acquired after the analysis. The standard values thus obtained provide a quantitative assessment of the relationship between the levels of biomarkers and disease status. This method enabled the evaluation of the effect of CRT on biomarker groups (Inflammation, Oxidative stress, Extracellular matrix remodeling, Neurohormones, Myocyte injury, Myocyte stress).

\section{Results}

A total of 44 patients (mean age $61.6 \pm 11.7$ years, 14 females) with successful implantation of biventricular implantable cardioverter defibrillators were included in the study. Out of these, $32(72.7 \%)$ patients were considered responders while the remaining $12(27.2 \%)$ patients were nonresponders, as they did not respond to CRT. Out of the total patients included, left bundle branch block was present in all $32(100 \%)$ responders and in $9(75 \%)$ non-responders. Another baseline demographic, electrocardiographic and echocardiographic parameters were comparable in both responders and the non-responders (Table 1). Considerable left ventricle reverse remodeling was seen in all the responders with a mean decrease in $\mathrm{LV}$ end-systolic volume index from $75.96 \pm 29.9$ $\mathrm{mL} / \mathrm{m}^{2}$ to $58.56 \pm 24.7 \mathrm{~mL} / \mathrm{m}^{2}(\mathrm{p}=0.001)$ and a mean increase in $\mathrm{LV}$ ejection fraction from $27.0 \pm 5.47 \%$ at baseline to $35.44 \pm 8.08 \%$ at 12 -month follow-up $(\mathrm{p}=0.001)$. While the $\mathrm{QRS}$ width showed a significant decrease in the responders from 162.5 (IQR 144-180) ms to 143 (IQR 130-158) ms ( $\mathrm{p}=0.001$ ), a non-significant increase was observed in the non-responders as well (145 [IQR 128-166] ms to 164 [IQR 130-186] ms, p = 0.533). 
Analysis of the responders showed that, at the 12-month follow-up visit, levels of IL-6, MPO, oxLDL, MMP-2, galectin-3, troponin T, and BNP were significantly lower than the baseline; while the levels of IL-4, hsCRP, TNF- $\alpha$, uric acid, MMP-9, prokol-3NT, endothelin-1, chromogranin A and CK-MB did not show a significant change. On the other hand, in the non-responders, the levels of MPO and MMP-2 were lower; uric acid level was higher and the levels of IL-4, IL-6, hsCRP, TNF- $\alpha$, oxLDL, MMP-9, prokol-3NT, galectin-3, endothelin-1, chromogranin A, troponin T, CK-MB, and BNP did not show a significant change at 12 -month follow-up (Table 2).

Out of the pathways involved in the pathogenesis of HF, significant changes were observed in three of them, i.e. myocyte stress, extracellular matrix (ECM) remodeling, and oxidative stress, of the six pathways that were studied. At follow-up, no significant changes were seen in the inflammatory, myocyte injury, and neurohormonal pathways. On repeated measures, two-way ANOVA showed that the changes in myocyte stress markers had the greatest effect size (effect size $=0.357 ; \mathrm{p}=0.001$ ) followed by changes in markers of ECM remodeling (effect size $=0.343 ; \mathrm{p}=0.015)$ and oxidative stress (effect size $=0.247 ; \mathrm{p}=0.039)($ Table 3$)$.

In the present study, out of 44 patients included, $33(75 \%)$ were clinical responders (improvement NYHA class $\geq 1$ ). When analysis was performed in the clinical responders on the basis of clinical improvement, a decrease in levels of IL-6, MPO, oxLDL, galectin-3, MMP-2, MMP-9, endothelin-1, troponin $\mathrm{T}$, and BNP was found while levels of IL-4, TNF- $\alpha$, hsCRP, uric acid, prokol-3NT, chromogranin A, and CK-MB did not show any change. Similar findings were obtained with ANOVA analysis, viz., surrogate biomarkers of oxidative stress, ECM remodeling, and myocyte stress showed a significant improvement in clinical responder patients. In the study population, 27 patients had non-ischemic etiology for HF. Of these, $22(81.5 \%)$ were found to be responders. Biochemical analysis in the patients with HF due to nonischemic etiology revealed that levels of IL-6, TNF- $\alpha$, MPO, MMP-2, galectin-3, and BNP decreased; while levels of IL-4, hsCRP, uric acid, oxLDL, MMP-9, prokol-3NT, endothelin-1, chromogranin $\mathrm{A}$, troponin $\mathrm{T}$ and CK-MB levels showed no improvement. When the biomarkers were considered as a pathway group, a significant improvement was observed in oxidative stress, ECM remodeling, and myocyte stress biomarker groups (data not shown).
For the prediction of CRT response, MPO, uric acid, oxLDL, chromogranin $\mathrm{A}$, and BNP were found to be potential covariates in the univariate analysis (MPO: odds ratio [OR] 0.999, 95\% confidence interval [CI] 0.998-1.001, $\mathrm{p}=0.206$; uric acid: OR 1.488, 95\% CI 0.975-2.273, $\mathrm{p}=0.066$; oxLDL: OR 0.960, 95\% CI 0.920-1.002, $\mathrm{p}=0.059$; chromogranin A: OR 0.997, 95\% CI 0.993-1.001, $\mathrm{p}=0.126$; BNP: OR 0.998, 95\% CI 0.997-0.999, $\mathrm{p}=0.016$, respectively). After adjustment for the covariates with multivariate regression analysis, it was found that preimplantation BNP and oxLDL were independent predictors of CRT response (BNP: OR 0.998, 95\% CI 0.997-0.999, $\mathrm{p}=0.042$; oxLDL: OR 0.956, 95\% CI 0.907-0.999, $\mathrm{p}=0.049$, respectively). The most sensitive BNP and oxLDL cut-off levels for CRT response determined by ROC analysis were $682 \mathrm{pg} / \mathrm{mL}$ and $37.5 \mathrm{ng} / \mathrm{mL}$, respectively. In order to further explore the relationships between the investigated biomarkers, a detailed correlation analysis was performed (Table 4). Troponin T levels correlated significantly with uric acid (rho $=0.495, \mathrm{p}=0.001$ ), MMP-2 $($ rho $=0.425, \mathrm{p}=0.010)$, galectin $-3($ rho $=0.329$, $\mathrm{p}=0.033$ ), and BNP (rho $=0.397, \mathrm{p}=0.009$ ). IL-4 levels correlated in turn with hsCRP (rho = $=0.321, \mathrm{p}=0.041)$, and inversely with CK-MB (rho $=-0.330, \mathrm{p}=0.035$ ). Furthermore, hsCRP levels correlated significantly with uric acid (rho $=$ $=0.358, p=0.025)$. MPO levels correlated significantly with galectin-3 (rho $=0.310, p=0.041)$. In addition, BNP and MMP-2 levels were found to be significantly correlated (rho $=0.457, \mathrm{p}=0.004)$.

\section{Discussion}

In the present study, it was found that CRT is associated with an improvement in the biomarkers of myocyte stress, ECM remodeling, and oxidative stress, besides having a beneficial effect on the clinical and echocardiographic parameters. The most prominent change was seen in the markers of myocyte stress, followed by those of oxidative stress and ECM remodeling. However, the biomarkers of inflammation, neurohormones, and myocyte injury did not show a statistically significant change.

Cardiac remodeling mainly results in cardiac dysfunction. It involves a pathophysiological substrate for its onset and progresses to ventricular dysfunction. Consequently, cellular and molecular changes occur, which result in a progressive loss of ventricular function and dilatation leading to QRS prolongation. QRS expansion leads to molecular 


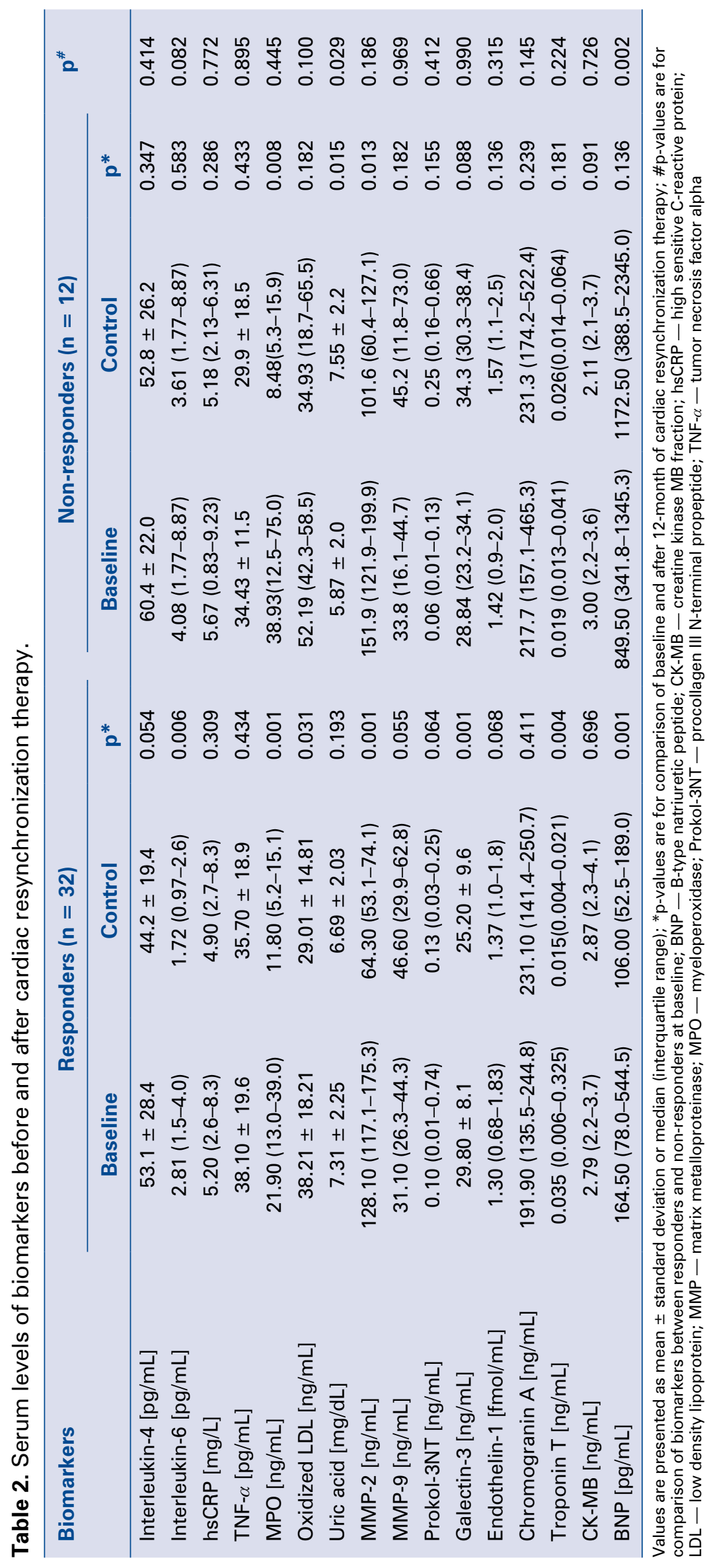


Table 3. Analysis of the changes in biomarkers after cardiac resynchronization therapy, one-by-one and as a group, by employing repeated measures of two-way analysis of variance.

\begin{tabular}{|c|c|c|c|c|}
\hline \multirow[t]{2}{*}{ Pathways and biomarkers } & \multicolumn{2}{|c|}{ Effect size (Partial Eta Squared) } & \multicolumn{2}{|r|}{$\mathbf{P}$} \\
\hline & Responders & Non-responders & Responders & Non-responders \\
\hline Inflammation & 0.111 & 0.096 & 0.100 & 0.414 \\
\hline Interleukin-4 & 0.115 & 0.047 & 0.054 & 0.459 \\
\hline Interleukin-6 & 0.194 & 0.003 & 0.01 & 0.858 \\
\hline hsCRP & 0.093 & 0.105 & 0.085 & 0.257 \\
\hline TNF- $\alpha$ & 0.042 & 0.230 & 0.251 & 0.083 \\
\hline Oxidative stress & 0.247 & 0.331 & 0.039 & 0.068 \\
\hline Myeloperoxidase & 0.508 & 0.357 & 0.001 & 0.024 \\
\hline Uric acid & 0.093 & 0.489 & 0.085 & 0.005 \\
\hline Oxidized LDL & 0.141 & 0.147 & 0.031 & 0.175 \\
\hline Extracellular-matrix remodeling & 0.343 & 0.358 & 0.015 & 0.060 \\
\hline MMP-2 & 0.677 & 0.637 & 0.001 & 0.001 \\
\hline MMP-9 & 0.125 & 0.148 & 0.044 & 0.174 \\
\hline Prokol-3NT & 0.168 & 0.307 & 0.018 & 0.04 \\
\hline Galectin-3 & 0.402 & 0.342 & 0.001 & 0.028 \\
\hline Neurohormonal pathways & 0.069 & 0.050 & 0.272 & 0.204 \\
\hline Endothelin-1 & 0.122 & 0.071 & 0.046 & 0.356 \\
\hline Chromogranin A & 0.015 & 0.277 & 0.498 & 0.053 \\
\hline Myocyte injury & 0.046 & 0.199 & 0.536 & 0.137 \\
\hline Troponin T & 0.091 & 0.122 & 0.088 & 0.221 \\
\hline CK-MB & 0.001 & 0.277 & 0.985 & 0.053 \\
\hline Myocyte stress (BNP) & 0.357 & 0.088 & 0.001 & 0.303 \\
\hline
\end{tabular}

BNP - B-type natriuretic peptide; CK-MB — creatine kinase MB fraction; hsCRP — high sensitive C-reactive protein; LDL — low density lipoprotein; MMP — matrix metalloproteinase; Prokol-3NT — procollagen III N-terminal propeptide; TNF- $\alpha$ - tumor necrosis factor alpha

polarization and re-arrangement of myocardial blood [13, 14]. The studies on the pathogenesis of HF have shown that in the process of cardiac dysfunction, inflammation, oxidative stress, ECM remodeling, neurohormones, myocyte damage, and myocyte stress play a role $[1,15]$. It is important to study the changes in the pathophysiological process after resynchronization between the ventricular walls.

Cardiac resynchronization therapy reduces $\mathrm{BNP}$ and troponin levels by decreasing intraventricular pressure and myocardial wall tension [16]. Comprehensive investigations have been carried out on the biological role and the potential clinical applications of the natriuretic peptides. Many studies have shown that a significant decrease in BNP levels occurs after CRT, resulting in a good prognosis [17]. The results obtained in this study are comparable to those of the previous studies. It has also been found that the positive effects of CRT on myocyte stress are accompanied by a decrease in BNP levels. Though a significant decrease was seen in myocyte stress, yet there were only limited changes seen in the biomarkers of myocyte damage after CRT. The results of the present study are consistent with those of previous research which has shown that an increase in ventricular wall tension results in much lower subclinical myocardial damage when compared to ischemic heart disease [18].

Ventricular remodeling plays a significant role in the progression to HF. The ECM provides a skeleton for myocytes and determines their size and shape. The ECM is a metabolically active structure with a continuous turnover of its elements so that a dynamic balance between the synthesis and degradation of collagen is maintained. Myofibroblasts function to regulate collagen turnover and respond to stimuli like a mechanical stretch, wall stress, autocrine, and paracrine factors. The response of the myofibroblasts to these factors results in an imbalance between MMPs and their inhibitors, which is in turn associated with ventricular dysfunction and dilatation [19]. Any abnormality in collagen metabolism may also 


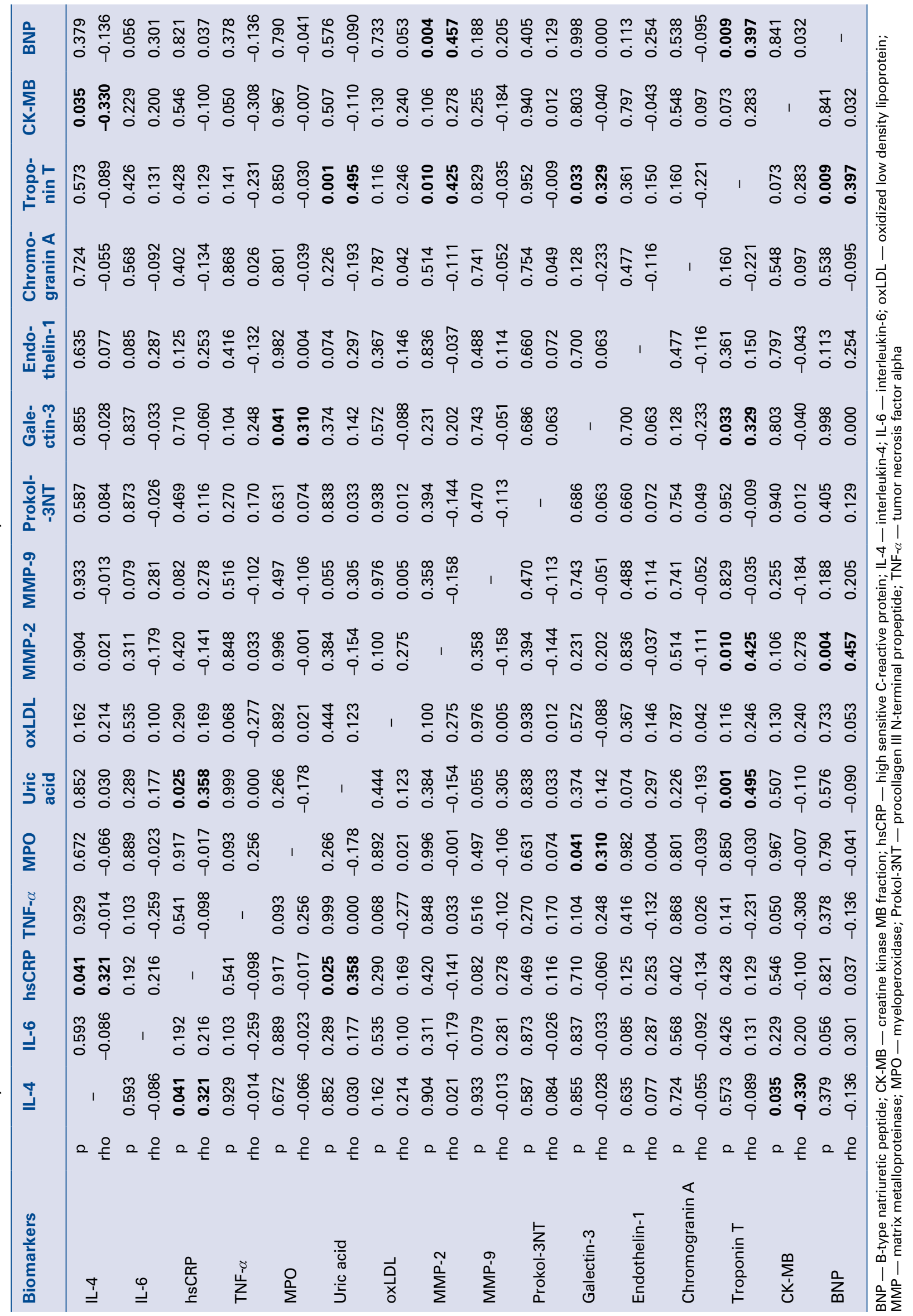


prove to be deleterious to cardiac functions as the resultant fibrotic changes can result in impairment of ventricular function [20]. Cardiac synchronization decreases the mechanical tension and stress imposed on the left ventricle. As the process of reverse remodeling begins, an improvement in ECM metabolism and a decrease in the levels of ECM remodeling markers (like galectin-3, prokol$-3 \mathrm{NT}$, and MMPs) is also seen [21, 22]. In the present study, a significant decrease was observed in the levels of MMP-9 and galectin-3. However, the changes in the levels of MMP-2 and procollagen were not significant. Some previous studies have also demonstrated that systemic MMP levels may not exactly reflect MMP metabolism in the cardiac muscle; while other studies have shown that an increase in collagen precursors may actually reflect reverse cardiac remodeling [23].

Oxidative stress results from an imbalance between the formations of reactive oxygen species (ROS) and endogenous antioxidant defense mechanisms. The imbalance in oxidative metabolism has deleterious effects on the progression to HF. Oxidative stress can damage cellular proteins and lead to myocyte apoptosis and necrosis. Since direct quantification of reactive oxygen species in humans is difficult, indirect markers of oxidative stress have been developed that include molecules like oxLDL, uric acid, and MPO. An increase in the level of plasma oxLDL in a peripheral blood sample may indicate an increase in ROS in the failing myocardium or in the poorly perfused peripheral muscles of patients with HF. Uric acid is a predictor of poor prognosis in patients with HF. During HF, the levels of circulating MPO increase and correlate with disease intensity $[15,24]$. On the contrary, the improvements in the clinical status of patients and reverse remodeling after resynchronization therapy are associated with a decrease in oxidative stress $[25,26]$. The levels of biomarkers indicating oxidative stress were improved at the end of the follow-up period in the present study, which is consistent with these findings. Another significant finding is that the oxLDL and BNP were found to be independent predictors for CRT response. Since the present study is the first of its kind investigating the oxLDL in the CRT patients, the findings of this study form a base for further studies.

It has been established that $\mathrm{HF}$ is associated with systemic inflammation and that it involves the interplay between pro-inflammatory and inhibitory cytokines. It is hypothesized that CRT reduces inflammation and that it may be one of the several factors responsible for improving the symptoms of
HF. However, the available literature reports conflicting results and suggests that the role of CRT in reducing inflammation is not uniform, rather it shows variations due to different interactions occurring during the disease process $[27,28]$. It was also found in the present study that no significant change occured in the levels of inflammatory biomarkers, except in IL-6.

It is well known that during HF the neurohormones increase as a response to a vasoconstriction due to reduced peripheral flow, and this response results in further myocardial damage while also worsening cardiac functions [29]. Though the effect of neurohormones in the pathogenesis of $\mathrm{HF}$ was first identified more than 50 years ago, its role in dyssynchrony remains unclear [1]. When the power of contraction of the heart decreases, the resultant decline in peripheral circulation induces an increase in catecholamine and endothelin levels in order to restore circulation. Previous studies have demonstrated that increased endothelin-1 levels are associated with both ventricular and vascular fibrosis. The level of chromogranin-A, which increases circulation may be associated with neuroendocrine activation and to the clinical severity in patients with HF [29-31]. However, no decrease in levels of endothelin-1 and chromogranin-A after CRT implantation was found in the present study, suggesting that the optimal medical therapy, particularly the beta-blockers, may affect the concentration of neurohormones in patients with HF.

\section{Limitations of the study}

This study had a few limitations, firstly, this was a small-sized preliminary and hypothesis generating study rather than a cause-effect association study. The small sample size especially poses difficulty in statistical analysis for the prediction of CRT response and the correlation between each biomarker. In addition, according to the current guidelines, class I recommendation has been introduced for CRT in patients with QRS duration $\geq 150 \mathrm{~ms}$ and this therapy is not recommended for the patients whose QRS duration is less than $130 \mathrm{~ms}$. However, only two-thirds of patients included in the present study met this criterion. Secondly, six pathophysiological pathways and more than 30 biomarkers are associated with both development and progression of HF. The pathways were investigated together in order to understand how they are affected by CRT therapy using a limited number of biomarkers. As the blood samples were obtained only at two points in time, i.e., at the time of initiation of the study and at 12 -month 
follow-up, it is also possible that some information on these markers may have been missed during the period in between. Thirdly, certain pathways are not independent but are interdependent. The inflammatory, oxidative and remodeling processes constitutes complex chemical reactions that involve many molecules; therefore eliminating an individual variation among the circulating biomarkers is almost impossible. In addition, some biomarkers may also be indicative of multiple pathophysiological pathways.

\section{Conclusions}

In the present study, a significant reduction was observed only in the biomarkers of myocardial stress, ECM remodeling and oxidative stress among all the CRT responder subjects. Nevertheless, further large-scale studies are required to confirm the results of this preliminary, hypothesis generating study.

\section{Acknowledgements}

This study was supported by a grant from the Turkish Society of Cardiology (No: 2010/1, Date: 2 June 2010).

\section{Conflict of interest: None declared}

\section{References}

1. Braunwald E. Biomarkers in heart failure. N Engl J Med. 2008; 358(20): 2148-2159, doi: 10.1056/NEJMra0800239, indexed in Pubmed: 18480207.

2. Kamireddy S, Agarwal SK, Adelstein E, et al. Correlation of electrical and mechanical reverse remodeling after cardiac resynchronization therapy. Ann Noninvasive Electrocardiol. 2009; 14(2): 153-157, doi: 10.1111/j.1542-474X.2009.00290.x, indexed in Pubmed: 19419400.

3. Karaahmet T, Tigen K, Mutlu B, et al. Prognostic significance of left ventricular systolic dyssynchrony in patients with nonischemic dilated cardiomyopathy. Turk Kardiyol Dern Ars. 2009; 37(5): 301-306, indexed in Pubmed: 19875901.

4. Versteeg H, Schiffer AA, Widdershoven JW, et al. Response to cardiac resynchronization therapy: is it time to expand the criteria? Pacing Clin Electrophysiol. 2009; 32(10): 1247-1256, doi: 10.1111/j.1540-8159.2009.02505.x, indexed in Pubmed: 19702599.

5. El Sabbagh FH, Guzon OJ, Alpert MA, et al. Electrocardiographic clues to identify nonresponders to cardiac resynchronization therapy. Ann Noninvasive Electrocardiol. 2010; 15(4): 369-377, doi: 10.1111/j.1542-474X.2010.00393.x, indexed in Pubmed: 20946560.

6. Balci KG, Balci MM, Sen F, et al. The role of baseline indirect inflammatory markers in prediction of response to cardiac resynchronisation therapy. Kardiol Pol. 2016; 74(2): 119-126, doi: 10.5603/KP.a2015.0142, indexed in Pubmed: 26202536.
7. Pitzalis MV, Iacoviello M, Di Serio F, et al. Prognostic value of brain natriuretic peptide in the management of patients receiving cardiac resynchronization therapy. Eur J Heart Fail. 2006; 8(5): 509-514, doi: 10.1016/j.ejheart.2005.10.013, indexed in Pubmed: 16503416.

8. García-Bolao I, Macías A, López B, et al. A biomarker of myocardial fibrosis predicts long-term response to cardiac resynchronization therapy. J Am Coll Cardiol. 2006; 47(11): 2335-2337, doi: 10.1016/j.jacc.2006.03.012, indexed in Pubmed: 16750706.

9. Gronda E, Genovese S, Padeletti L, et al. Renal function impairment predicts mortality in patients with chronic heart failure treated with resynchronization therapy. Cardiol J. 2015; 22(4): 459-466, doi: 10.5603/CJ.a2015.0019, indexed in Pubmed: 26325259 .

10. Morales MA, Maltinti M, Piacenti M, et al. Adrenomedullin plasma levels predict left ventricular reverse remodeling after cardiac resynchronization therapy. Pacing Clin Electrophysiol. 2010; 33(7): 865-872, doi: 10.1111/j.1540-8159.2010.02723.x, indexed in Pubmed: 20230463.

11. Karaca O, Omaygenc MO, Cakal B, et al. Adjusting the QRS duration by body mass index for prediction of response to cardiac resynchronization therapy: does one QRS size fit all? Ann Noninvasive Electrocardiol. 2016; 21(5): 450-459, doi: 10.1111/ anec.12346, indexed in Pubmed: 26820486.

12. Pitzalis MV, Iacoviello M, Romito R, et al. Cardiac resynchronization therapy tailored by echocardiographic evaluation of ventricular asynchrony. J Am Coll Cardiol. 2002; 40(9): 1615-1622, indexed in Pubmed: 12427414.

13. Kashani A, Barold SS. Significance of QRS complex duration in patients with heart failure. J Am Coll Cardiol. 2005; 46(12): 2183-2192, doi: 10.1016/j.jacc.2005.01.071, indexed in Pubmed: 16360044.

14. Cho H, Barth AS, Tomaselli GF. Basic science of cardiac resynchronization therapy: molecular and electrophysiological mechanisms. Circ Arrhythm Electrophysiol. 2012; 5(3): 594-603, doi: 10.1161/CIRCEP.111.962746, indexed in Pubmed: 22715238.

15. Emdin M, Vittorini S, Passino C, et al. Old and new biomarkers of heart failure. Eur J Heart Fail. 2009; 11(4): 331-335, doi: 10.1093/eurjhf/hfp035, indexed in Pubmed: 19329823.

16. Sugiura T, Takase H, Toriyama T, et al. Circulating levels of myocardial proteins predict future deterioration of congestive heart failure. J Card Fail. 2005; 11(7): 504-509, doi: 10.1016/j. cardfail.2005.04.025, indexed in Pubmed: 16198245.

17. Shalaby AA, Abraham WT, Fonarow GC, et al. Association of BNP and troponin levels with outcome among cardiac resynchronization therapy recipients. Pacing Clin Electrophysiol. 2015; 38(5): 581-590, doi: 10.1111/pace.12610, indexed in Pubmed: 25677851.

18. Pastormerlo LE, Agazio A, Benelli E, et al. Usefulness of highsensitive troponin elevation after effort stress to unveil vulnerable myocardium in patients with heart failure. Am J Cardiol. 2015; 116(4): 567-572, doi: 10.1016/j.amjcard.2015.05.017, indexed in Pubmed: 26089013.

19. López B, González A, Díez J. Circulating biomarkers of collagen metabolism in cardiac diseases. Circulation. 2010; 121(14): 1645-1654, doi: 10.1161/CIRCULATIONAHA.109.912774, indexed in Pubmed: 20385961.

20. Chalikias GK, Tziakas DN. Biomarkers of the extracellular matrix and of collagen fragments. Clin Chim Acta. 2015; 443: 39-47, doi: 10.1016/j.cca.2014.06.028, indexed in Pubmed: 25007952. 
21. Stolen CM, Adourian A, Meyer TE, et al. Plasma galectin-3 and heart failure outcomes in MADIT-CRT (multicenter automatic defibrillator implantation trial with cardiac resynchronization therapy). J Card Fail. 2014; 20(11): 793-799, doi: 10.1016/j.cardfail.2014.07.018, indexed in Pubmed: 25106783.

22. Stanciu AE, Vatasescu RG, Stanciu MM, et al. Cardiac resynchronization therapy in patients with chronic heart failure is associated with anti-inflammatory and anti-remodeling effects. Clin Biochem. 2013; 46(3): 230-234, doi: 10.1016/j.clinbiochem.2012.11.002, indexed in Pubmed: 23159290.

23. Peterson JT, Hallak H, Johnson L, et al. Matrix metalloproteinase inhibition attenuates left ventricular remodeling and dysfunction in a rat model of progressive heart failure. Circulation. 2001; 103(18): 2303-2309, indexed in Pubmed: 11342481.

24. von Lueder TG, Girerd N, Atar D, et al. Serum uric acid is associated with mortality and heart failure hospitalizations in patients with complicated myocardial infarction: findings from the High-Risk Myocardial Infarction Database Initiative. Eur J Heart Fail. 2015; 17(11): 1144-1151, doi: 10.1002/ejhf.419, indexed in Pubmed: 26424212.

25. Krupa W, Rozwodowska M, Sielski S, et al. Influence of cardiac resynchronization therapy on oxidative stress markers in patients with chronic heart failure. Cardiol J. 2014; 21(5): 576-582, doi: 10.5603/CJ.a2014.0051, indexed in Pubmed: 25471244.

26. Michowitz Y, Kisil S, Guzner-Gur H, et al. Usefulness of serum myeloperoxidase in prediction of mortality in patients with severe heart failure. Isr Med Assoc J. 2008; 10(12): 884-888, indexed in Pubmed: 19160948.

27. Bose A, Truong QA, Singh JP. Biomarkers in electrophysiology: role in arrhythmias and resynchronization therapy. J Interv Card Electrophysiol. 2015; 43(1): 31-44, doi: 10.1007/s10840-0159982-7, indexed in Pubmed: 25715916.

28. Belperio J, Horwich T, Abraham WT, et al. Inflammatory mediators and clinical outcome in patients with advanced heart failure receiving cardiac resynchronization therapy. Am J Cardiol. 2016; 117(4): 617-625, doi: 10.1016/j.amjcard.2015.11.049, indexed in Pubmed: 26832186.

29. Menardi E, Vado A, Rossetti G, et al. Cardiac resynchronization therapy modifies the neurohormonal profile, hemodynamic and functional capacity in heart failure patients. Arch Med Res. 2008; 39(7): 702-708, doi: 10.1016/j.arcmed.2008.07.004, indexed in Pubmed: 18760200.

30. Boriani G, Regoli F, Saporito D, et al. Neurohormones and inflammatory mediators in patients with heart failure undergoing cardiac resynchronization therapy: time courses and prediction of response. Peptides. 2006; 27(7): 1776-1786, doi: 10.1016/j. peptides.2006.02.010, indexed in Pubmed: 16621149.

31. Dong YX, Burnett JC, Chen HH, et al. Effect of cardiac resynchronization therapy on broad neurohormone biomarkers in heart failure. J Interv Card Electrophysiol. 2011; 30(3): 241-249, doi: 10.1007/s10840-011-9551-7, indexed in Pubmed: 21336616. 